\title{
Relaciones intercultural
La experiencia del circo y su singularidad
}

Recibido: 19 de julio, 2018.

Aceptado: 12 de octubre, 2020.

Por: Dra. Sylvia Contreras-Salinas, Universidad de Santiago de Chile, Chile, ORCID: 0000-0003-2297-2399, y Dra. Mónica Ramírez-Pavelic, Universidad Arturo Prat de Iquique, Chile, ORCID: 0000-0003-2710-2982.

\section{Resumen}

Este artículo busca apreciar la configuración de relaciones interculturales desde una perspectiva crítica. A este respecto, se considera que el circo es un buen ejemplo para precisar cuestiones que muchas veces quedan en la abstracción, e impiden la acción comunicativa y la implicancia en la generación de los mundos sociales. El objetivo fue describir las acciones comunicativas y las prácticas de ciudadanía que construyen los integrantes del circo Markoning. La metodología utilizada corresponde al enfoque cualitativo; específicamente, a un estudio de caso, el cual fue abordado a través del análisis del discurso. Los principales hallazgos ilustran los desafíos y señalan un escenario propicio para indagar acerca de cómo se van gestando las relaciones interculturales a nivel local, situado y cotidiano. Como conclusión, se menciona que la acción comunicativa permite reconocer la singularidad del circo, mientras que sus prácticas de ciudadanía dan cuenta de una legítima participación periférica en el espacio público.

\section{Abstract}

\section{Intercultural relations: The circus experience and its uniqueness}

This article gives an account of some key elements of a research focused on members of a Chilean family circus. The study seeks to appreciate the construction of intercultural relations from a critical perspective. In this regard, the circus serves to clarify issues that often remain in abstraction, thus preventing communicative action and impacting the generation of social worlds. The objective was to describe the communicative actions and citizenship practices that build the members of the Markoning circus. The methodology used corresponds to the qualitative approach, specifically a case study, which was approached utilizing discourse analysis. The main findings illustrate the challenges in the field of social sciences and point to an appropriate scenario to inquire how intercultural relations are constructed at a local, situated, and daily level. We conclude that communicative action allows to recognize the distinctive social capital of the circus, while citizenship practices reaffirm their option of not appearing in the public space.

1 La doctora Sylvia Contreras-Pavelic es profesora del Departamento de Educación de la Universidad de Santiago de Chile. Contacto: sylvia.contreras.s@usach.cl.

2 La doctora Mónica Ramírez-Pavelic es docente e investigadora de la Universidad Arturo Prat de Iquique, Chile. Contacto: trapecio@gmail.com.

PALABRAS CLAVE:

circo, artes escénicas, pluralismo cultural, prácticas, nomadismo, diálogo, diversidad cultural.

\section{KEY WORDS:}

circus, performing arts, cultural pluralism, practices, nomadism, dialogue, cultural diversity. 


\section{INTRODUCCIÓN}

La experiencia del circo y su singularidad resultan relevantes para problematizar múltiples supuestos, tanto en torno al diálogo de saberes, como en el marco de la interculturalización y la ciudadanización. A partir de ello, se aprecia la condición de pluralidad del ser humano, en la iniciativa de legitimar los saberes que han sido olvidados o excluidos en el lado oculto de la modernidad. En este contexto, la diversidad de valoración entre los seres humanos queda clara en los planteamientos de Arendt (2005), "debido a que todos somos lo mismo, es decir, humanos, y por tanto nadie es igual a cualquier otro que haya vivido, viva o vivirá" (p. 22).

Se entiende que la pluralidad mencionada previamente, se visualiza en la acción, condición inevitablemente política de posicionamiento entre los seres humanos que se manifiesta "en el pluriculturalismo, donde "lo pluri" evidencia típicamente una convivencia de culturas en el mismo espacio territorial, aunque sin una profunda interrelación equitativa" (Walsh, 2011, p. 140). De este modo, la interculturalidad se torna en la meta que se desea alcanzar para tener sociedades muy distintas a las presentes, donde cada ser humano aparezca ante los otros por la:

voluntad de actuar y hablar, de insertar el propio yo en el mundo y comenzar una historia personal (...) donde el valor e incluso la audacia se encuentran ya presentes al abandonar el lugar oculto y privado y mostrar quien es uno, al revelar y exponer el propio yo (Arendt, 2005, p. 210).

En esta empresa, el estado asumiría un rol coercitivo que muchas veces da la espalda a la condición de pluralidad/diversidad, así como a la legítima demanda de las personas en definirse como propietarias de los bienes sociales, lo cual limita, además, la comprensión de la ciudadanía como una condición bidireccional del ciudadano a la comunidad y de la comunidad al ciudadano (Cortina, 2009). En este sentido, se destacan las palabras de Bolívar (2016), quien señala que el aprendizaje del oficio de ciudadano, de manera situada, debe realizarse en el espacio donde viven las personas, pero ampliarse a otros ámbitos y prácticas que marquen su vida cotidiana.

De este modo, en el espacio público el discurso representa "la realización de la condición humana de la pluralidad, es decir, de vivir como ser distinto y único entre iguales" (Arendt, 2005, p. 202). Es decir, un discurso que se re-crea con otros en busca de la conformación de una vida plena. Para esto, los seres humanos deben apelar a una "ciudadanía y unos derechos que no hablan únicamente de la estructura formal de una sociedad; sino que, además, indican el estado de la lucha por el reconocimiento de los otros como sujetos de intereses válidos, valores pertinentes y demandas legítimas" (Cortina, 2009, p. 98). Al respecto, se entiende la ciudadanía como práctica de descolonización de las instancias que compartimos con los más cercanos.

Desde esta visión, el concepto de ciudadanía puede parecer contradictorio y limitado, pues al fundarse en la idea de igualdad/diferencia, acerca a los semejantes y separa de los diferentes. En esta línea, debe mencionarse la distinción entre lo público y privado, donde cada persona está llamada a implicarse en los asuntos públicos a través de la argumentación y la deliberación, es decir, mediante la socialidad (Cortina, 2009). Una situación que resulta pertinente frente a las demandas actuales, ya que, de este modo, las sociedades, estados o naciones, solo pueden cobrar significado en la relevancia con la acción reflejada en el discurso.

Por tanto, se entiende la ciudadanía como la práctica que cobra sentido en un espacio en donde las personas se organizan para interactuar. Es una acción que se sostiene desde la premisa de la pluralidad/diversidad e insta a una ética intercultural que:

no se contenta con asimilar las culturas relegadas a la triunfante, ni siquiera con la mera coexistencia de las culturas, sino que invita a un diálogo, de forma que respeten sus diferencias y vayan dilucidando conjuntamente que consideran irrenunciable para construir desde todas ellas una convivencia más justa y feliz (Cortina, 2009, p. 183).

En definitiva, se propone un diálogo intercultural.

Se considera que una de las instancias en las que se aprecian algunos elementos de pluralidad/diversidad es en los circos familiares que deambulan aún por diversos países del mundo. En ellos, se encarna la idea de pluralidad/diversidad y el diálogo intercultural busca abrirse paso, pues "la experiencia circen- 
se constituye una realidad social significativa que se presenta como ícono del acervo cultural" (Contreras, 2010, p. 99).

Esta investigación, permite adentrarse en el enigmático mundo del circo, a través de una familia que recorre los espacios rurales chilenos. Ellos comparten parte de su historia, prácticas de socialización, estigmatización, identidad y nomadismo, entre otras características. En esta línea, se aprecia cómo el circo construye espacios de comunicación entre sus miembros, quienes mantienen relaciones duraderas, pero se vinculan limitadamente con los demás, mediante el ejercicio de una ciudadanía diversa. En síntesis, esta investigación se adentra en las acciones comunicativas y las particulares prácticas de ciudadanía que conforman los integrantes del circo en estudio.

\section{METOdOLOGÍA}

El estudio se orientó con base en las siguientes interrogantes: ¿cómo definen los integrantes del circo la propiedad personal y comunal?, ¿cuál es la relación que establecen con la familia y la comunidad?, ¿Cómo constituyen sus procesos de integración/estigmatización?, ¿cómo definen su trashumancia?

El presente proyecto fue llevado a cabo en varias etapas. Primero, una fase exploratoria-preparatoria que consistió en el establecimiento de contacto y entrevistas con diversos tipos de circos: familiares, de empresa, circo-show, entre otros; además de entrevistas con miembros del sindicato de artistas circenses. Segundo, una revisión documental y encuentro personal con la familia Rozas. Tercero, la elaboración del análisis y la redacción del informe. Entre los instrumentos utilizados, caben mencionar: las entrevistas, las historias de vida y la observación participante.

La metodología utilizada corresponde al enfoque cualitativo; específicamente, a un estudio de caso que fue analizado a la luz del análisis de discurso. Para efectos del trabajo de investigación, los discursos se codificaron y categorizaron en un proceso inductivo propio de esta perspectiva. Estos discursos fueron originados a partir de prácticas lingüísticas conformadas en las relaciones sociales, cuyo análisis se realiza a partir de la actuación de dichas prácticas en el presente (De Lucas, Izquierdo, Jiménez y Celayeta, 2015). En suma, al entenderse el análisis de discurso como método y perspectiva, se concep- tualiza la producción discursiva como una práctica social que permite la comprensión y el estudio de los procesos sociales que constituyen la esencia del circo Markoning.

Los objetivos de esta investigación fueron planteados desde la perspectiva sociológica tradicional; es decir, buscando describir y comprender un fenómeno particular constituido por un actor social que transita en los territorios rurales de Chile: el circo familiar. Específicamente, la investigación busca describir las acciones comunicativas y las prácticas de ciudadanía que construyen los integrantes del circo Markoning.

Los siete integrantes del circo, con edades comprendidas entre 17 y 74 años, aceptaron participar voluntariamente en la investigación. Para esto, se realizaron al menos tres entrevistas narrativas a cada uno de ellos. Además, se recogió la información de historias de vida y observación participante. Los datos obtenidos fueron transcritos y organizados con base en códigos y categorías.

\section{RESULTADOS Y DISCUSIÓN}

\section{El rescate de la historia}

El circo Markoning fue estableciendo sus propios espacios y construyendo su propia carpa y carromatos:

Cuando empezaron los circos mi papá empezó trabajando en galpones o corredores, casi la mayoría de ellos eran cirqueros, gente que se inició en el circo, empezó así, por ser mi papá trabajaba de artista, después ya como le quedaban debiendo o no le pagaban... empezaron a trabajar solos él y mi mamá, ellos buscaban galpones y ahí trabajan, mi mamá hacia un número que se colgaba del pelo, casi tocaba el suelo, pero igual hacían el número, mi papá hacia trapecio, hacia el toni, hacia un número que tragaba espadas. Él empezó de a poquitito a hacer el circo, primero tenía una galería chiquitita de cuatro tablas y una carpa chiquitita (Rozas, 2003a).

En este extracto, se aprecia cómo se constituye una forma concreta de propiedad personal y comunal, en donde las redes familiares se visualizan como la base de la comunidad circense, la cual es extrapolada cuando cada miembro de la familia se vincula con otro circo a través de los lazos familiares. 
Así, el circo en Chile es representado como una gran familia que se mueve por el territorio. Cada circo se concibe como una familia, cuyas funciones tienden a permanecer indiferenciadas dentro de ella, pues existe una alta homogeneidad debido a que el cambio es regido por la tradición y la herencia: "al final en el circo todos somos familia" (Rozas, 2003b). En esta línea, la familia corresponde al grupo social constitutivo del circo Markoning (y de la mayoría de los circos en Chile): "al final todos somos conocidos, casi todos familiares, por algún lado hay algún parentesco" (Rozas, 2003b).

Esta condición de parentesco y cercanía permite que las personas de circo pienses, sobretodo, en el bienestar de todos los artistas circenses: "uno también tiene que pensar en el circo de allá, porque al final todos somos iguales" (Rozas, 2003c). Tal situación demuestra que los miembros del circo establecen fuertes vínculos comunitarios y que muestran una identidad colectiva eminentemente circense (Contreras, 2010). Principios que se encuentran registrados en la sabiduría Andina descrita por Rengifo (2003), en donde se alude a "prácticas que se fundan en el vínculo, como lazos que eslabonan y anudan a cada uno de los seres" (p. 55).

\section{Prácticas de socialización}

En este entorno, los principales hallazgos se mezclan con las prácticas circenses, que, en los discursos de los miembros, se enuncian como: "No conozco otra forma de vida" (Rozas, 2003b); "Aprendí el número porque mi mamá me enseño" (Rozas, 2003a). Al respecto, queda claro que la socialización responde a patrones muy tradicionales, propios de una comunidad que da cuenta de una relación cara a cara e intergeneracional, puesto que sustenta la reproducción de procesos elementales al interior del circo.

\section{Entorno social}

Las relaciones con el entorno social se desprenden de los discursos: "Somos personas que no molestan a nadie y que nadie nos moleste a nosotros" (Rozas, 2003c), "Con la gente de afuera no tengo mucho contacto" (Rozas, 2003a), "Ahora votamos, pero todos en blanco, porque no nos gusta" (Rozas, 2003a). Entonces, la carpa marca las fronteras del mundo circense con los otros mundos, en donde media el mínimo contacto entre ellos. En tal sentido, dicho contacto está movido por intereses estratégicos e instrumentales; es decir, un mundo que se constituye dentro de otro mundo de carácter hegemónico, que obliga a cumplir ciertas prácticas bajo la falsa ilusión de una ciudadanía.

En otras palabras, "la interacción con el exterior sólo se da a través del espectáculo. Las relaciones humanas que se construyen en torno al trabajo marcan formas de pensar, sentir y actuar que se transmiten en el proceso de socialización" (Contreras, 2010, p. 107).

\section{¿Qué es el circo?}

Frente a esta pregunta, los entrevistados señalan: "O sea... es su vida" (Rozas, 2003b), "el circo es la misma casa" (Rozas, 2003c), "El circo entra por la vista" (Rozas, 2003c), "El circo es de los niños" (Rozas, 2003b) y "El circo es veleidoso" (Rozas, 2003a). A partir de dichas afirmaciones, el circo se 'profesa', es decir, adopta un carácter sagrado que conforma una representación omni-abarcante. En otras palabras, constituye la representación que impregna todas las demás representaciones, mientras que como símbolo glorificado de la cotidianidad confiere máxima importancia al cuidado de la imagen que proyecta al exterior; particularmente, para atraer a los niños y a las niñas.

\section{Estigmatización}

La estigmatización se aprecia en algunas de las afirmaciones mencionadas por el entrevistado: "Hay gente que mira en menos a los de circo" (Rozas, 2003a), "en el campo la gente se alegra cuando llega un circo... pero en las poblaciones o pueblos grandes sale la gente, le niegan el agua..." (Rozas, 2003b), "De repente nos miran como pobrecitos" (Rozas, 2003a). En la actualidad, el circo es más valorado en las zonas rurales, debido a que una especie de solidaridad de clase parece ponerse en juego. Sin embargo, el ser pobres los deslegitima e impide su aparición en el espacio público (Arendt, 2005). En palabras de Escobar (2003), la comunidad circense es excluida, deslegitimada y olvidada en la construcción de una sociedad moderna, y se visualiza como su lado oscuro. Para Cortina (2017), lo cierto es que las puertas se cierran ante las personas pobres, aquellos "que no tienen que perder más que sus cadenas (...) condenados mundialmente a la invisibilidad" (p. 86). 


\section{Nomadismo}

Sobre el nomadismo, Rozas (2003b) menciona que "Uno tiene que pensar que es circo, que va volviendo, de todas partes se va volviendo...". La trashumancia es una propiedad que define al circo y marca intensamente el proceso de socialización, ya que le confiriere una diferencia esencial con otras formas de vida. Así, los miembros del circo nacen, viven y envejecen en una suerte de presente intenso, en donde poco cabe la preocupación por lo que les depara el futuro. Cada vez que se llega a un pueblo, surgen nuevas expectativas y la vida parece recomenzar desde ese lugar. El espectáculo que se muestra al nuevo público renueva el modo de ver y sentir el mundo. Este es el espacio que no se abandona (Contreras, 2010).

\section{Identidad}

Los discursos en torno a la identidad, visibilizan algunas representaciones: "todos nos vemos igual... buenos artistas" (Rozas, 2003a) y "somos cirqueros" (Rozas, 2003b). Con la autodefinición de "cirqueros", ellos tipifican tanto su propia situación dentro del mundo social del circo, como las diversas relaciones que tienen con sus semejantes y con los objetos culturales. Ser "cirquero" significa que cada uno es capaz de realizar todas las tareas que implica el circo y manejar todos los saberes necesarios para realizar dichas actividades. Esta tipificación ensambla convenientemente con las necesidades del circo Markoning.

\section{Su aparecer en el ámbito público}

Según Ibáñez (2014), la suma de saberes cotidianos que nos brinda la gente de circo, es la suma de los saberes sobre la realidad que utilizan de modo efectivo en su vida cotidiana.

Al responder la pregunta ¿Qué es el circo?, René responde "es su vida" (Rozas, 2003c). Al respecto, el circo se convierte en una realidad empírica por derecho propio. Representa una vivienda: "o sea, el circo es la misma casa" (Rozas, 2003b), un lugar propio. Pero, además, el circo es movilidad y renovación:

Es aquí donde usted ve distintas cosas, en el circo nunca es la misma carpa, uno o dos años y después hay otra. ¿Entiende? claro, el circo se va renovando, los pueblos son distintos, en el circo va viendo otras cosas (Rozas, 2003b).
Asimismo, es una oportunidad de diversión, una entretención destinada primordialmente a los pequeños: "el circo es de los niños, tendrían que acabarse los niños en el mundo como para que el circo muriera" (Rozas, 2003b). De esta forma, el circo desarrolla un espectáculo que encierra muchas acciones atrayentes e introduce en la ilusión y en el vértigo que destruye la estabilidad de la percepción y aparta de la vida común gracias a sus números artísticos, su carpa y sus vestimentas.

\section{El circo siempre vuelve}

El peligro de desaparecer no pasa solo por la ausencia de espectadores, sino por la irresponsabilidad de sus miembros. "O solo que lo mate uno mismo" (Rozas, 2003c). Al conformar el circo una representación colectiva que los actores construyen, estos mismos pueden destruirla al no existir intersubjetividad, reciprocidad de perspectivas, intercambio de puntos de vista o una congruencia de definiciones relevantes y parecidas sobre lo que es y debe ser el circo. $\mathrm{Al}$ respecto, Georgina menciona: "Pero no piensan que es circo y que tiene que volver el próximo año" (Rozas, 2003a), puesto que el circo debe preocuparse por establecer una relación honesta con la gente.

No obstante, debido a los problemas que se les presentan en el camino, la falta de terrenos para instalarse, dificultades para conseguir agua y luz, y la declinación del entusiasmo por los espectáculos circenses, la gente de circo indica que su vida no es fácil: "el circo es muy difícil... hay algunos que creen que es otra cosa el circo" (Rozas, 2003b).

\section{La diferencia}

Asimismo, los integrantes del circo Markoning perciben que las otras personas actúan de un modo que los hace sentir diferentes: "lo miran distinto" (Rozas, 2003c), lo cual conforma el rótulo "gente de circo", "saben que uno es de circo" (Rozas, 2003a). En este marco, los habitantes de los lugares donde se asienta el circo actúan de dos modos diferentes; por una parte, algunos muestran cierta aceptación por su llegada: "No nos tratan mal" (Rozas, 2003b), mientras que, por otra parte, algunos manifiestan abiertamente su desagrado: "como que la gente le tiene mala a los de circo" (Rozas, 2003a). "Hay gente que nos trata de "circo pobre" (Rozas, 2003c). 
Con base en lo anterior, se visualizan estereotipos vertidos bajo la mirada de sociedades que, en general, han evolucionado hacia un nivel de organización muy planificada. Situación que refuerza la creencia de que, para ser feliz, se debe consumir una determinada cantidad y tipo de bienes y servicios: "de repente nos miran como pobrecitos y nos preguntan: ¿ustedes no pasan frio ahí?, yo les digo: ¿por qué? ¿Y esos carritos no son helados? y uno tiene que decir que son igual que una casa" (Rozas, 2003a). En este contexto, se releva la "urgencia de hacer dialogar más que culturas, -intra-inter- y alter culturalidad, civilizaciones con sus propias cosmovisiones y lógicas de vida/multidiálogo" (Yampara, 2006, p. 35).

En consonancia, se considera relevante desmontar la representación de la periferia como la personificación del retraso, e identificar individuos capaces de transformar su realidad. Para ello, se "debe comenzar desde los estratos más humildes, convirtiendo al ser humano en sujeto protagónico de su historia" (Alvarado, 2015, p. 105). Esto con el propósito de evitar, de este modo, la justificación de diversos procesos de transportación global de la razón y el progreso, que han llevado a pensamientos excluyentes y desiguales, bajo el supuesto de pretender objetividad y neutralidad. Lo anterior conlleva a que, poco a poco, se eliminen las redes que sujetan a los seres humanos, lo que exige que se avance en la solidaridad y en un conocimiento-reconocimiento (De Santos-Sousa, 2003).

\section{CONCLUSIÓN}

El circo se transforma en un buen ejemplo para precisar cuestiones que muchas veces quedan en la abstracción e impiden la acción comunicativa y la implicancia en la generación de los mundos sociales; puesto que este demanda una comprensión que se integre en un marco heurístico y que tenga la capacidad de visibilizar la utopía de la construcción de la igualdad en la diferencia. Es decir, conformar una pertenencia bajo la lógica del saber, que deje atrás el conocimiento, sin olvidar que la pluralidad es una condición que se revela en la medida en que la interculturalización y la ciudadanización aparecen en las esferas públicas y privadas.

En suma, la realidad del circo ofrece la posibilidad de constituir espacios de comunicación con el fin de generar un mundo social de la vida intercultural. Por ello, los miembros del circo Markoning, con sus relaciones permanentes, prolongadas y de carácter subjetivo, se encuentran siempre expuestos a intercambios con los grupos sociales que los rodean. Así, el circo debe intercambiar con el público en el montaje de su espectáculo, pero además interactuar con la sociedad y con sus instituciones para obtener insumos o bienes básicos.

De este modo, al relacionarse en otros escenarios, los cirqueros se percatan de que no requieren una mayor internalización de modelos, pues ellos se identifican con un otro generalizado, construido dentro de las fronteras del circo. Por tanto, ya no se tiene que recorrer grandes distancias para encontrar una comunidad que vive su vida de forma distinta a la que impera en nuestras sociedades. En este sentido, el circo nos ofrece un rico escenario comunicativo, que puede entregar pistas sobre la construcción de sociedades plurales, lo cual deja atrás la idea de interculturalidad cosmopolita que se concibe en la lejanía.

El ejemplo del circo ofrece también la posibilidad de profundizar y reflexionar en torno a la práctica de la ciudadanía. Por cuanto este ha manifestado su no aparición en el espacio público, por lo que conforma una colectividad que no participa en la vida pública, es decir, no salen de la periferia, de la exclusión, de su carpa, para darse a conocer. ¿Por qué no lo hacen, por qué eligen estar ocultos y aislados en su carpa? Estas son preguntas que solo serán respondidas en una acción comunicativa, en la generación de un saber en el que participen ellos y otros. Con base en "la voluntad de actuar y hablar, de insertar el propio yo en el mundo y comenzar una historia" (Arendt, 2005, p. 210).

Asimismo, dicha acción comunicativa nos permite enriquecer las miradas en torno a la familia: ¿de qué manera, en la actualidad, familias que recorren pueblos y ciudades modernas constituyen un capital social, el aval para nuevas relaciones que marcan, además, un sello distintivo? Cada circo tiene su nombre, su carpa, arregla sus propios asuntos, tiene un lenguaje distintivo y una variedad de formas simbólicas que se van construyendo en las relaciones familiares y que se transmiten de una generación a otra para demostrar que son distintos a los demás. En esta 
línea, "la familia es el 'todo' que conforma el 'ser de circo' y que se completa mediante la evolución de una realidad a otra o elevando ambas realidades: familia y circo a una forma superior" (Contreras, 2010).

Conjuntamente, el circo promueve un proceso de socialización que limita el horizonte social de sus miembros al disminuir sus intercambios con el mundo externo, por cuanto rodea de cerca a sus asociados, manteniéndolos fuertemente ligados a su medio cotidiano y, por consiguiente, a sus tradiciones. Situación que asegura que no se filtren elementos extranjeros que puedan provocar divergencias. A esta tarea, contribuye el hecho de establecer límites concretos y definidos: el espacio que ocupa la carpa en conjunto con los carromatos.

Se considera que la realidad mencionada debe profundizarse en la lógica de la interculturalidad, para abrir nuevas líneas de investigación, pues, ciertamente, el circo Markoning conforma una comunidad, con la idea de una obligación entre quienes viven bajo su carpa. La persona que nace, crece y vive en los límites de este circo, está claramente sujeta al dominio de los otros miembros en su convivir habitual. El arbitrio de los otros dispone las posibilidades cotidianas de cada uno, lo cual ofrece posibilidades de estudios en torno a la autonomía, desde un pensamiento liberal y a la ética de la alteridad.

Para Ibáñez (2014), la realidad del circo nos muestra lo perturbado que pueden estar los contactos interpersonales. Asimismo, las relaciones que mantiene la gente de circo con su entorno señalan lo alienado de una sociedad. Está claro que no se puede apreciar la totalidad de las relaciones interpersonales del grupo o de la comunidad en donde están situados, pero probablemente "toda relación personal refleja algo de la naturaleza de la totalidad social" (Heller, 2002, p. 345). Por tanto, también se visualiza un campo de estudio en torno a la cotidianidad; así como a la relación entre los conocimientos científicos y los saberes cotidianos que se verifican desde abajo, desde las necesidades sociales y personales (Heller, 2002).

Otra línea de investigación relacionada con las anteriores, considera que muchos agentes, entre ellos el circo, no buscan integrarse, sino, más bien, participar de un espacio más amplio donde puedan desarrollar su proyecto y reconstruir su vivencia para convertirla en una experiencia cultural. Para, de esta manera, mostrar formas alternativas al pensamiento hegemónico, que no pretende fundamentar sino, por el contrario, busca poner de manifiesto la caducidad de algunas formas de vida (Contreras y Ramírez, 2017; Vattimo, 2013).

En suma, el circo constituye un amplio escenario para la investigación aplicada u otras en la línea de la legítima participación periférica. Se vislumbra como un campo rico de investigación para las ciencias sociales; en especial, desde la interpelación de la interculturalidad, la pluralidad, la ciudadanía y el saber.

\section{REFERENCIAS BIBLIOGRÁFICAS}

Alvarado, J. (2015). Pensar la educación en clave decolonial. Revista de Filosofía, 81(3), 103-116.

Arendt, H. (2005). La condición humana. Barcelona: Paidós Ibérica

Bolívar, A. (2016). Educar democráticamente para una ciudadanía activa. Revista Internacional de educación para la justicia social, 5(1), 69-87.

Contreras, S. y Ramírez, M. (2017). Análisis fenomenológico del tropo "pasar a llevar". Revista Atenea (515), 203219. Recuperado de https://dx.doi.org/10.4067/S071804622017000100203

Contreras, S. (2010). El circo: un encadenamiento de sentido. Revista Atenea, 502, 97-109.

Cortina, A. (2009). Ciudadanos del mundo. Hacia una teoría de la ciudadanía. Madrid: Editorial Alianza.

Cortina, A. (2017). Aporofobia, el rechazo al pobre: Un desafío para la sociedad democrática. Barcelona: Paidós.

De Lucas, A., Izquierdo, D., Jiménez, F., Celayeta, N. (2015). Aplicaciones y enfoques teóricos del análisis del discurso. Navarra: EUNSA Ediciones.

De Sousa-Santos, B. (2003). Crítica de la razón indolente. Contra el desperdicio de la experiencia. Volumen 1. Bilbao: Editorial Desclée.

Escobar, A. (2003). Mundos y conocimientos de otro modo. El programa de investigación de modernidad/colonialidad latinoamericano. Tabula Rasa, 1, 51-86.

G. Rozas, comunicación personal, noviembre 2003a.

Heller, A. (2002). Sociología de la vida cotidiana. Barcelona: Ediciones Península.

Ibañez, J. (2014). Por una sociología de la vida cotidiana. Buenos Aires: Siglo XXI. 
Relaciones interculturales: La experiencia del circo y su singularidad

M. Rozas, comunicación personal, noviembre 2003b.

R. Rozas, comunicación personal, noviembre 2003c.

Rengifo, G. (2003). La enseñanza de estar contento. Educación y afirmación cultural andina. Lima: Ediciones PRATEC.

Vattimo, G. (2013). El sentido de la existencia: posmodernidad y nihilismo. Bilbao: Publicaciones de la Universidad de Deusto.

Walsh, C. (2011). Interculturalidad, plurinacionalidad y decolonialidad: las insurgencias político-epistémicas de refundar el Estado. Tabula Rasa, 9, 131-152.

Yampara, S. (2006). Cosmovisión, Uruq-Pacha, desarroIlo y/o Qamaña Andino. En F. Delgado y J.C. Mariscal (Eds.), Educación intra e intercultural. Alternativas a la Reforma Educativa Neocolonizadora (pp. 23-36). La Paz: Agruco-Compas. 